\title{
The Stability of Certain Vector Bundles on Multi-Projective Spaces
}

\author{
E. Ballico ${ }^{1}$ \\ Dept. of Mathematics \\ University of Trento \\ 38050 Povo (TN), Italy \\ ballico@science.unitn.it
}

\begin{abstract}
Here we study the stability of certain homogeneous vector bundles on multi-projective spaces using their restriction to certain rational curves.
\end{abstract}

Mathematics Subject Classification: 14J60; 14N05

Keywords: multi-projective space; stable vector bundle; homogeneous vector bundle

\section{INTRODUCTION}

Fix integers $k \geq 2, n_{i}>0,1 \leq i \leq k$, and an algebraically closed base field $\mathbb{K}$. Set $\Pi:=\mathbf{P}^{n_{1}} \times \cdots \times \mathbf{P}^{n_{k}}$. Let $e_{i} \in \mathbb{Z}^{k}$ denote the vector $(0, \ldots, 1, \ldots, 0)$ in which 1 appears in the $i$-th coordinate. Set $V_{i}:=H^{0}\left(\Pi, \mathcal{O}_{\Pi}\left(e_{i}\right)\right)$. Hence $\operatorname{dim}\left(V_{i}\right)=n_{i}+1$. Let $\rho: \oplus_{i=1} V_{i} \otimes \mathcal{O}_{\Pi}\left(e_{i}\right) \rightarrow \mathcal{O}_{\Pi}$ be the evaluation map. Set $\Lambda:=\operatorname{Ker}(\rho)$. Hence we have an exact sequence

$$
0 \rightarrow \Lambda \rightarrow \oplus_{i=1} V_{i} \otimes \mathcal{O}_{\Pi}\left(e_{i}\right) \stackrel{\rho}{\rightarrow} \mathcal{O}_{\Pi} \rightarrow 0
$$

These vector bundles are important for the following reason. For any closed subscheme $X \subset \Pi$ the cohomology groups $h^{i}\left(\Pi, \mathcal{I}_{X} \otimes\left(a_{1}, \ldots, a_{k}\right)\right), i=0,1$, are strongly related to the existence and number of " essential " generators in multi-degree $\left(a_{1}, \ldots, a_{k}\right)$ of the multi-homogeneous ideal of $T$. In the case $k=1$ we have $\Lambda=\Omega_{\mathbf{P}^{n_{1}}}^{1}$ and the relation is well-known and heavily used to study the minimal free resolution of $X$. See [3] and references therein for several results and questions on the generators of the multi-homogeneous ideal when $X$ is a finite set. Hence it is natural to study the vector bundle $\Lambda$. Here we will study its stability with respect to the polarizations of $\Pi$ (see Theorem 1 and Corollary 1) and the restriction of $\Lambda$ to certain rational curves (see Proposition 1). $\Lambda$ is homogeneous for the action of the connected group

\footnotetext{
${ }^{1}$ The author was partially supported by MIUR and GNSAGA of INdAM (Italy).
} 
$\prod_{i=1}^{k} P G L\left(n_{i}\right)=\operatorname{Aut}^{0}(\Pi)$. If $n_{i}=n_{j}$ for some $i \neq j$, then it is also invariant with respect to the exchange of these two factors. Hence it is easy to check that $\Lambda$ is homogeneous for the action of Aut(П). Fix integers $h_{i}>0,1 \leq i \leq k$, and set $H:=\mathcal{O}_{\Pi}\left(h_{1}, \ldots, h_{k}\right)$. Here we will consider slope $H$-stability and call it $H$-stability. It is easy to check that (assuming $k \geq 2$, as always in this paper unless otherwise stated) the $H$-stability of $\Lambda$ may depend from $H$ (Remarks 2 and 3). We will also consider the following more general set-up. Fix integers $d_{i}>0,1 \leq i \leq k$, and set $V_{i, d_{i}}:=H^{0}\left(\Pi, \mathcal{O}_{\Pi}\left(d_{i} e_{i}\right)\right)$. Hence $\operatorname{dim}\left(V_{i, d_{i}}\right)=$ $\left(\begin{array}{c}n_{i}+d_{i} \\ n_{i}\end{array}\right)$. Let $\rho_{\left[d_{1}, \ldots, d_{k}\right]}: \bigoplus_{i=1} V_{i} \otimes \mathcal{O}_{\Pi}\left(-d_{i} e_{i}\right) \rightarrow \mathcal{O}_{\Pi}$ be the evaluation map. Set $\Lambda_{\left[d_{1}, \ldots, d_{k}\right]}:=\operatorname{Ker}\left(\rho_{\left[d_{1}, \ldots, d_{k}\right]}\right)$. The map $\rho_{\left[d_{1}, \ldots, d_{k}\right]}$ is surjective and the vector bundle $\Lambda_{\left[d_{1}, \ldots, d_{k}\right]}$ is homogeneous for the action of the connected group $\operatorname{Aut}^{0}(\Pi)$. We have $\operatorname{det}\left(\Lambda_{\left[d_{1}, \ldots, d_{k}\right]}\right) \cong \mathcal{O}_{\Pi}\left(-\sum_{i=1}^{k}\left(\begin{array}{c}n_{i}+d_{i} \\ n_{i}\end{array}\right) e_{i}\right)$. Now take linear subspaces $W_{i} \subseteq V_{i}, 1 \leq i \leq k$, such that the evaluation map $\rho_{\left[d_{1}, \ldots, d_{k} ; W_{1}, \ldots, W_{k}\right]}: \oplus_{i=1} W_{i} \otimes$ $\mathcal{O}_{\Pi}\left(-d_{i} e_{i}\right) \rightarrow \mathcal{O}_{\Pi}$ is surjective. Set $\Lambda_{\left[d_{1}, \ldots, d_{k} ; W_{1}, \ldots, W_{k}\right]}:=\operatorname{Ker}\left(\rho_{\left[d_{1}, \ldots, d_{k} ; W_{1}, \ldots, W_{k}\right]}\right)$.

Proposition 1. Fix integers $k \geq 2, x>0, d_{i}>0, x_{i}, n_{i}>0, m_{i}>0$, $1 \leq i \leq k$, such that $x_{i} d_{i}=x_{j} d_{j}$ for all $i, j$ and $1 \leq m_{i} \leq\left(\begin{array}{c}n_{i}+x \\ n_{i}\end{array}\right)$ for all $i$. Fix general linear subspaces $W_{i} \subseteq H^{0}\left(\mathbf{P}^{n_{i}}, \mathcal{O}_{\mathbf{P}^{n_{i}}}\left(x_{i}\right)\right)$ such that $\operatorname{dim}\left(W_{i}\right)=m_{i}$ for all $i$. Set $u:=\sum_{i=1}^{k} m_{i}-1, a=\left\lfloor\left(u k x_{1} d_{1}\right) / u\right\rfloor$ and $e:=k x_{1} d_{1}-a u$. Let $u=\left(u_{1}, \ldots, u_{n}\right): \mathbf{P}^{1} \rightarrow \Pi$ be a general morphism of multi-degree $\left(d_{1}, \ldots, d_{k}\right)$. Then $u^{*}\left(\Lambda_{\left[d_{1}, \ldots, d_{k} ; W_{1}, \ldots, W_{k}\right]}\right)$ has splitting type $a_{1} \geq \cdots \geq a_{u}$ with $a_{i}=a+1$ for $1 \leq i \leq e$ and $a_{i}=a$ for $e+1 \leq i \leq u$.

Theorem 1. Fix an integer $x>0$ and assume $h_{i}=h_{j}$ for all $i \neq j$. Then $\Lambda_{[x, \ldots, x]}$ is $H$-stable.

The case $x=1$ of Theorem 1 is the following result.

Corollary 1. Assume $h_{i}=h_{j}$ for all $i, j$. Then $\Lambda$ is $H$-stable.

\section{THE PROOFS}

Proofs of Proposition 1. The statement means that $u^{*}\left(\Lambda_{\left[d_{1}, \ldots, d_{k} ; W_{1}, \ldots, W_{k}\right]}\right)$ is rigid. Since rigidity is an open condition, both $W_{i}$ and $u_{i}$ are general and $x_{i} d_{i}=x_{j} d_{j}$ for all $i, j$, it is sufficient to prove the existence of an exact sequence of vector bundles on $\mathbf{P}^{1}$

$$
0 \rightarrow A \rightarrow \mathcal{O}_{\mathbf{P}^{1}}\left(-x_{1} d_{1}\right)^{\oplus u} \rightarrow \mathcal{O}_{\mathbf{P}^{1}} \rightarrow 0
$$

with $A$ rigid. The existence of the exact sequence (2) is a very particular case of $[2]$.

Fix $i \in\{1, \ldots, k\}$. Let $\pi_{i}: \Pi \rightarrow \mathbf{P}^{n_{i}}$ denote the projection on the $i$-th factor, $\Pi_{i}$ the product of all factors of $\Pi$, except the $i$-th and $\eta_{i}: \Pi \rightarrow \Pi_{i}$ the projection.

Remark 1. Let $E$ be a vector bundle on $\Pi$. Fix $i \in\{1, \ldots, k\}$, any fiber $T \equiv \mathbf{P}^{n_{i}}$. We have $\Lambda \mid T \equiv \Omega_{T}^{1} \oplus \mathcal{O}_{T}^{x}, x:=k-1+\sum_{j \neq i} n_{j}$. If $E \mid D$ is trivial for 
every line $D \subseteq T$, then $E \mid T$ is trivial. If this is true for all $T$ and all $i$, then $E$ is trivial.

There is a natural $\operatorname{Aut}^{0}(\Pi)$-invariant map $\alpha: \oplus_{i=1}^{k} \pi_{i}^{*}\left(\Omega_{\mathbf{P}^{n_{1}}}^{1}\right) \rightarrow \Lambda$. The restriction of $\alpha$ to any fiber of $\eta_{1}$ shows that $\alpha$ is injective and with locally free cokernel. Thus there is an exact sequence on $\Pi$

$$
0 \rightarrow \oplus_{i=1}^{k} \pi_{i}^{*}\left(\Omega_{\mathbf{P}^{n_{i}}}^{1}\right) \rightarrow \Lambda \rightarrow M \rightarrow 0
$$

in which $M$ is a rank $k-1$ vector bundle on $\Pi$ which is homogeneous for the action of $\operatorname{Aut}^{0}(\Pi)$. We have $\operatorname{det}(G) \equiv \mathcal{O}_{\Pi}$ and $M \mid D$ is trivial for every line $D$ contained in a fiber of $\eta_{i}$. Remark 1 gives that $M$ is trivial, i.e. we have the exact sequence

$$
0 \rightarrow \oplus_{i=1}^{k} \pi_{i}^{*}\left(\Omega_{\mathbf{P}^{n_{i}}}^{1}\right) \rightarrow \Lambda \stackrel{f}{\rightarrow} \mathcal{O}_{\Pi}^{\oplus(k-1)} \rightarrow 0
$$

Remark 2. Recall that $\operatorname{det}(\Lambda) \equiv \mathcal{O}_{\Pi}\left(-\sum_{i=1}^{k}\left(n_{i}+1\right) e_{i}\right)$. Fix an integer $i$ such that $1 \leq i \leq k$. If $h_{i}\left(n_{i}+1\right) / n_{i}<\left(\sum_{j \neq i} h_{j}\left(n_{j}+1\right)\right) /\left(\sum_{j \neq i} n_{j}+k-1\right)$ (resp. $\left.h_{i}\left(n_{i}+1\right) / n_{i} \leq\left(\sum_{j \neq i} h_{j}\left(n_{j}+1\right)\right) /\left(\sum_{j \neq i} n_{j}+k-1\right)\right)$, then the inclusion $\pi_{i}^{*}\left(\Omega_{\mathbf{P}^{n_{i}}}^{1}\right) \rightarrow \Lambda$ associated to (3) shows that $\Lambda$ is not $H$-semistable (resp. not $H$-stable).

Remark 3. Fix integers $k \geq 2$ and $n_{i}>0,1 \leq i \leq k$. Fix $i \in\{1, \ldots, k\}$ and an integer $h_{i}>0$. If $h_{j} \gg g_{i}$ for all $j \neq i$, then Remark 2 shows that $\Lambda$ is not $H$-semistable.

Proof of Theorem 1. Taking an equivalent polarization we reduce to the case in which $h_{i}=1$ for all $i$. Notice that $\mu_{H}\left(\Lambda_{[x, \ldots, x]}\right)=-x\left(k+\sum_{i=1}^{k} n_{i}\right) /(k-$ $\left.1+\sum_{i=1}^{k} n_{i}\right)$. Assume that $\Lambda_{[x, \ldots, x]}$ is not $H$-stable and take a proper subsheaf $G$ of $\Lambda_{[x, \ldots, x]}$ with maximal $H$-slope and (among these subbundles) with maximal rank. Set $s:=\operatorname{rank}(G)$ and $\mathcal{O}_{\Pi}\left(b_{1}, \ldots, b_{k}\right):=\operatorname{det}(G)$. By assumption we have $\left(b_{1}+\cdots+b_{k}\right) / s \geq-\left(k+\sum n_{i}\right) /\left(k-1+\sum n_{i}\right)$. Set $d:=k-1+\sum_{i=1}^{k} n_{i}$. Let $u=\left(u_{1}, \ldots, u_{k}\right): \mathbf{P}^{1} \rightarrow \Pi$ be the general morphism of multi-degree $(d, \ldots, d)$. Proposition 1 give that $u^{*}\left(\Lambda_{[x, \ldots, x]}\right)$ is semistable and with splitting type $-x k-\sum_{i=1}^{k} x n_{i}$. Since $h_{i}=h_{j}$ and $\operatorname{deg}\left(u^{*}\left(\mathcal{O}_{\Pi}\left(e_{i}\right)\right)=d=\operatorname{deg}\left(u^{*}\left(\mathcal{O}_{\Pi}\left(e_{j}\right)\right)\right.\right.$ for all $i, j$, we immediately get that $\Lambda_{[x, \ldots, x]}$ is semistable, that $b_{i}=b_{1}$ for all $i$ and that $x\left(k-1+\sum_{i=1}^{k} n_{i}\right) b_{1}=x s\left(k+\sum_{i=1}^{k} n_{i}\right)$. Since $\left(k-1+\sum_{i=1}^{k} n_{i}\right)$ and $\left(k+\sum_{i=1}^{k} n_{i}\right)$ are coprime and $1 \leq s<\left(k-1+\sum_{i=1}^{k} n_{i}\right)$, we got a contradiction.

\section{REFERENCES}

[1] E. Ballico, Generators for homogeneous ideal of $s$ general points in $\mathbb{P}^{3}$, J. Algebra 106 (1987), no. 1, 46-52.

[2] E. Schlesinger, Extensions of vector bundles on $\mathbb{P}^{1}$, Comm. Algebra 28 (2000), no. 12, $5833-5889$. 
[3] A. Van Tuyl, The defining ideal of a set of points in muti-projective space, J. London Math. Soc. (2) 72 (2005), no. 1, 73-90.

Received: August 30, 2007 